\title{
Pressure support ventilation-pro decreases propofol consumption and improves postoperative oxygenation index compared with pressure-controlled ventilation in children undergoing ambulatory surgery: a randomized controlled trial \\ Le mode de ventilation en aide inspiratoire PSVPRO réduit la consommation de propofol et améliore l'indice d'oxygénation postopératoire comparativement à la ventilation en pression contrôlée chez les enfants subissant une chirurgie ambulatoire : une étude randomisée contrôlée
}

\author{
Swapnabharati Moharana, MD • Divya Jain, MD • Neerja Bhardwaj, MD • \\ Komal Gandhi, MD $\cdot$ Sandhya Yaddanapudi, MD $\cdot$ Badal Parikh, MD
}

Received: 8 April 2019/Revised: 9 October 2019/Accepted: 23 October 2019/Published online: 2 January 2020

(C) Canadian Anesthesiologists' Society 2020

\begin{abstract}
Purpose The PSVPro mode is increasingly being used for surgeries under laryngeal mask airway owing to improved ventilator-patient synchrony and decreased work of breathing. We hypothesized that PSVPro ventilation mode would reduce consumption of anesthetic agents compared with pressure control ventilation (PCV).

Methods Seventy children between three and eight years of age undergoing elective lower abdominal and urological surgery were randomized into PCV group $(n=35)$ or PSVPro group $(n=35)$. General anesthesia was induced with sevoflurane and a Proseal $L M A^{T M}$ was inserted. Anesthesia was maintained with propofol infusion to maintain the entropy values between 40 and 60. In the $P C V$ mode, the inspiratory pressure was adjusted to obtain an expiratory tidal volume of $8 \mathrm{~mL} \cdot \mathrm{kg}^{-1}$ and a respiratory rate of 12-20/min. In the PSVPRO group, the flow trigger was set at $0.4 \mathrm{~L} \cdot \mathrm{min}^{-1}$ and pressure support was adjusted
\end{abstract}

Presentation: A part of the paper was presented at 66th annual conference of Indian Society of Anaesthesiologists held at Agra, India on November 2018, and received the best paper award.

S. Moharana, MD · D. Jain, MD ( $\bowtie) \cdot$ N. Bhardwaj, MD • K. Gandhi, MD · S. Yaddanapudi, MD · B. Parikh, MD Department of Anesthesia and Intensive Care, Postgraduate Institute of Medical Education and Research, Sector-12, Chandigarh 160012, India

e-mail: jaindivya77@rediffmail.com to obtain expiratory tidal volume of $8 \mathrm{~mL} \cdot \mathrm{kg}^{-1}$. Consumption of anesthetic agent was recorded as the primary outcome. Emergence time and discharge time were recorded as secondary outcomes.

Results The PSVPro group showed significant reduction in propofol consumption compared with the PCV group (mean difference, $33.3 \mu \mathrm{g}^{-1} \cdot \mathrm{kg}^{-1} \cdot \mathrm{min}^{-1}$; $95 \%$ confidence interval [CI], 24.2 to 42.2). There was decrease in the emergence time in the PSVPro group compared with the PCV group (mean difference, $3.5 \mathrm{~min} ; 95 \% \mathrm{CI}, 2.8$ to 4.2) and in time to achieve modified Aldrete score $>9$ (mean difference, $3.6 \mathrm{~min}$; 95\% CI, 1.9 to 5.2).

Conclusion The PSVPro mode decreases propofol consumption and emergence time, and improves oxygenation index in children undergoing ambulatory surgery.

Trial registration Clinical Trial Registry of India (CTRI/ 2017/12/010942); registered 21 December, 2017.

Résumé

Objectif Le mode PSVPRO® est de plus en plus utilisé pour les chirurgies sous masque laryngé car il permet une meilleure synchronisation ventilateur-patient et réduit le travail respiratoire. Nous avons émis l'hypothèse que le mode de ventilation PSVPRO réduirait la consommation d'agents anesthésiques comparativement à une ventilation en pression contrôlée (VPC). 
Méthode Soixante-dix enfants âgés de trois à huit ans subissant une chirurgie abdominale ou urologique basse non urgente ont été randomisés en deux groupes, soit le groupe VPC $(n=35)$ et le groupe PSVPRO $(n=35)$. L'anesthésie générale a été induite à l'aide de sévoflurane et un masque laryngé Proseal $L M A^{T M}$ a été inséré. L'anesthésie a été maintenue par une perfusion de propofol afin de conserver des valeurs d'entropie entre 40 et 60. Dans le groupe VPC, la pression inspiratoire a été ajustée pour obtenir un volume télé-expiratoire de 8 $\mathrm{mL} \cdot \mathrm{kg}^{-1}$ et une fréquence respiratoire de 12-20/min. Dans le groupe PSVPRO, le seuil de détection du débit a été réglé à $0,4 \mathrm{~L} \cdot \mathrm{min}^{-1}$ et l'aide inspiratoire a été ajustée afin d'obtenir un volume télé-expiratoire de $8 \mathrm{~mL} \cdot \mathrm{kg}^{-1}$. Le délai jusqu'au réveil et jusqu'au congé ont été enregistrés comme critères secondaires.

Résultats Dans le groupe PSVPRO, une réduction significative de la consommation de propofol a été observée comparativement au groupe VPC (différence moyenne, $33,3 \mu \mathrm{g}^{-1} \cdot \mathrm{kg}^{-1} \cdot \mathrm{min}^{-1}$; intervalle de confiance [IC] $95 \%, 24,2$ à 42,2). Une réduction dans le délai pour le réveil a été observée dans le groupe PSVPRO par rapport au groupe VPC (différence moyenne, 3,5 min; IC $95 \%, 2,9$ à 4,2) et dans le délai jusqu'à l'obtention d'un score d'Aldrete modifié > 9 (différence moyenne, 3,6 min; IC $95 \%, 1,9$ à 5,2).

Conclusion Le mode PSVPRO réduit la consommation de propofol et le temps jusqu'au réveil tout en améliorant l'indice d'oxygénation chez les enfants subissant une chirurgie ambulatoire.

Enregistrement de l'étude Clinical Trial Registry of India (CTRI/2017/12/010942); enregistrée le 21 décembre 2017.

Unassisted spontaneous ventilation (SV) had been the conventional mode of ventilation with supraglottic airway devices (SAD) for ambulatory surgical practices. Nevertheless, the use of SV in longer surgeries is limited by inadequate ventilation, ineffective gaseous exchange, and fatigue resulting in hypoventilation. ${ }^{1}$

The advent of second-generation SADs like the LMA Proseal $^{\text {TM }}$ (Teleflex Medical, Morrisville, NC, USA) with higher sealing pressure and inbuilt gastric channel significantly increased the use of positive-pressure ventilation with SADs. ${ }^{2}$ As a result, the use of these SADs with pressure control ventilation (PCV) mode was compatible with longer surgeries, overcoming the limitations of $\mathrm{SV}^{3-5}$

Pressure support ventilation (PSV), initially used for weaning patients from ventilator support in the intensive care unit, has now been incorporated in the newer workstations in the operating theatre. ${ }^{6}$ It is a type of SV mode that senses the patient's inspiratory effort and delivers pressure support to overcome the resistance of the machine, breathing circuit, and airway device resulting in larger tidal volume than the patient could produce alone. $^{7}$ The pressure support synchronized with the respiratory effort of the patient results in better ventilator-patient synchrony and decreased work of breathing. ${ }^{8,9}$ Thus, this mode offers combined advantages of both PCV and SV at the same time.

The PSVPro ${ }^{\circledR}$ (GE Healthcare, Madison, WI, USA) mode is a subset of the PSV mode and provides machine delivered breaths should the patient's spontaneous ventilation slow or cease. This ventilation mode is upgraded in newer machines like Aestiva/5 (GE Healthcare, Madison, WI, USA).

In the current scenario, both PSV and PCV are being used as ventilation modes with SAD devices in ambulatory surgeries. ${ }^{5,10}$ We hypothesized that PSVPro will not only support the SV of the patient but also permit better patientventilator synchrony resulting in decreased anesthetic drug consumption and better oxygenation. We designed a randomized-controlled trial to compare the PSVPro and PCV modes of ventilation in unparalyzed children during ambulatory surgery under general anesthesia (GA) with the LMA Proseal. The primary objective was to compare consumption of anesthetic agent between PSVPro and PCV mode of ventilation. The secondary objective was to compare the SAD removal time, emergence time, and discharge time between these two ventilation modes. Oxygenation index and occurrence of asynchrony events were reported as exploratory outcomes.

\section{Methods}

The present prospective, randomized-controlled study was conducted in the advanced pediatric centre of a tertiary care hospital of North India after institutional ethics committee approval was given (ethics committee no: INT/IEC/2017/1157, 23 October, 2017 under Chairmanship of Professor K.L. Gupta) and written informed consent from the legal guardians of the children was obtained. The study was registered in the Clinical Trial Registry of India before the first patient was recruited (CTRI/2017/12/010942).

\section{Inclusion and exclusion criteria}

Seventy children with American Society of Anesthesiologists (ASA) physical status I and II between three and eight years of age undergoing elective lower 




Figure Consort flow diagram

abdominal and urological surgery lasting more than $45 \mathrm{~min}$ and less than two hours under GA were enrolled. Patients with anticipated difficult airway, emergency procedures, or surgical duration $<45$ min were excluded from the trial.

\section{Anesthesia protocol}

After premedication with $0.5 \mathrm{mg} \cdot \mathrm{kg}^{-1}$ of oral midazolam $30 \mathrm{~min}$ before surgery, children were taken to the operating room. Standard ASA monitors in the form of electrocardiography, pulse oximetry, and non-invasive blood pressure were used. In addition, depth of anesthesia was monitored using entropy module (GE Healthcare, Helsinki, Finland). General anesthesia was induced with 6-8 \% sevoflurane with oxygen. Fentanyl 2 $\mu \mathrm{g} \cdot \mathrm{kg}^{-1}$ was injected at the time of induction after securing intravenous access. Appropriate size LMA Proseal was inserted after confirming adequate depth of anesthesia. Acetaminophen $15 \mathrm{mg} \cdot \mathrm{kg}^{-1} i v$ and caudal block with 0.75
$\mathrm{mL} \cdot \mathrm{kg}^{-1}$ ropivacaine $0.2 \%$ were given to all the children after induction of anesthesia.

Anesthesia was maintained with propofol infusion and $60 \%$ nitrous oxide in oxygen. Propofol infusion was started initially at the rate of $200 \mu \mathrm{g} \cdot \mathrm{kg}^{-1} \cdot \mathrm{min}^{-1}$ and was titrated intraoperatively to maintain the entropy values between 40 and 60.

Study protocol

Children were randomly allocated using computer generated random number tables to the PCV or PSVPro group and ventilation was initiated as per group allocation through sequentially labelled opaque envelopes. These sealed opaque envelopes were opened by the anesthesiologist in charge of the case, who was not part of the study. Children in the PCV group were ventilated with PCV mode at a respiratory rate of 12-20 min and positive end-expiratory pressure (PEEP) of $4 \mathrm{~cm} \mathrm{H}_{2} \mathrm{O}$. The 
inspiratory pressure was set to achieve expired tidal volume (VTe) of $8 \mathrm{~mL} \cdot \mathrm{kg}^{-1}$. In the PSVPro group, ventilation was initiated once SV was present. In case of apnea after anesthesia induction, the child was manually ventilated till the $\mathrm{SV}$ regained. The flow trigger was set at $0.4 \mathrm{Lmin}^{-1}$ and pressure support was set to achieve VTe of $8 \mathrm{~mL} \cdot \mathrm{kg}^{-1}$ without PEEP.

Arterial blood gas was analyzed twice, once after the initiation of respective ventilatory modes and once at the time of skin closure. The oxygenation index was calculated as the ratio of partial pressure of arterial oxygen and fraction of inspired oxygen $\left(\mathrm{PaO}_{2} / \mathrm{F}_{\mathrm{I}} \mathrm{O}_{2}\right)$. Partial pressure of $\mathrm{CO}_{2}$ in arterial blood $\left(\mathrm{PaCO}_{2}\right)$ and $\mathrm{pH}$ were noted. All intraoperative asynchronous events were noted. We recorded ineffective breath generated by the patient during PCV mode, mainly arising because of inadequate depth of anesthesia as an asynchronous event. The episodes of ventilator asynchrony were treated by increasing the infusion rate of propofol by $50 \mu \mathrm{g} \cdot \mathrm{kg}^{-1} \cdot \mathrm{min}^{-1}$.

At the completion of surgery (on skin closure), propofol infusion was discontinued. The LMA Proseal was removed after spontaneous and regular respiration, satisfactory minute ventilation, and more than $95 \%$ oxygen saturation $\left(\mathrm{SpO}_{2}\right)$ were resumed. Any intraoperative complication, like laryngospasm, bronchospasm, desaturation, or dislodgement of LMA, was noted. In the postoperative period, all patients were transferred to the postanesthesia care unit (PACU). The data on propofol consumption, emergence time, oxygenation index, or any adverse events was noted by the anesthesiologist blinded to the group allocation.

\section{Study objectives}

The primary objective was to compare consumption of anesthetic agent between PSVPro and PCV mode of ventilation. The secondary objective was to compare the emergence time, SAD removal time, and discharge time between these two ventilation modes. Oxygenation index and occurrence of asynchrony events were reported as exploratory outcomes.

Time to removal of SAD was not reported separately (although it was initially registered as the secondary outcome) because the SAD was removed on complete emergence from anesthesia, and emergence time and SAD removal were similar in all the study patients. The discharge time was removed as a measured outcome because the children were discharged from the PACU after one to two hours of observation, despite fulfilling the discharge criteria according to the institutional protocol. Nevertheless, time to achieve a modified Aldrete score (MAS) $\geq 9$ was noted.

The anesthetic consumption was defined as the amount of propofol consumed from the start of infusion to the end of surgery (skin closure). Emergence time was defined as the time from stoppage of propofol infusion to spontaneous eye opening and response to verbal command. The entropy value response entropy (RE) and state entropy ( $\mathrm{SE}$ ) were monitored continuously and recorded at five time points (T0 = baseline, $\mathrm{T} 1=$ ten minutes after anesthesia induction, $\mathrm{T} 2=$ at surgical incision, $\mathrm{T} 3=$ at stoppage of propofol infusion, T4 = when fully awake). Any adverse events like LMA dislodgement, desaturation $<92 \%$, bronchospasm, and laryngospasm were also noted.

\section{Statistical analysis}

A sample of 33 patients in each group was calculated from the results of a previous study by Capdevila et al. ${ }^{10}$ who reported 852 (330) $\mathrm{mg}$ of propofol consumption in controlled mode compared with 610 (180) $\mathrm{mg}$ in a pressure support group. Therefore, at an alpha error of 0.05 and a power of $90 \%$ and allowing for approximately $10 \%$ dropouts, we aimed to recruit 35 patients in each group for this study.

The statistical analysis was carried out using Statistical Package for Social Sciences (version 22; SPSS Inc, Chicago, IL, USA). All quantitative variables were analyzed using measures of central tendency and measures of dispersion. Normality of data was checked by Lilliefors' test modification of the KolmogorovSmirnov test. The normally distributed data were compared using Student's $t$ test. For skewed data, MannWhitney U test was applied. The categorical data were analyzed using Chi square test or Fisher exact test as appropriate. A $P$ value $<0.05$ was considered significant.

\section{Results}

Ninety-two children were assessed for eligibility out of which 16 did not meet the inclusion criteria and six declined consent. A total of 70 children were randomized and analyzed (Figure). The demographic and intraoperative data were comparable between the two groups (Table 1).

There was a significant decrease in the propofol consumption in the PSVPro group (mean difference, 33.3 $\mu \mathrm{g}^{-1} \cdot \mathrm{kg}^{-1} \cdot \mathrm{min}^{-1} ; 95 \% \mathrm{CI}, 24.2$ to 42.2 ). There was a significant decrease in emergence time in the PSV group compared with the PCV group (mean difference, $3.5 \mathrm{~min}$; 95\% CI, 2.8 to 4.2 ) (Table 2).

There was significant difference in the time to achieve MAS $\geq 9$ (mean difference, $3.6 \mathrm{~min} ; 95 \% \mathrm{CI}, 1.9$ to 5.2 ) (Table 2).

Entropy, measured in the form of RE and SE was maintained between 40 and 60 intraoperatively. It was comparable in both groups at all time intervals (Table 2). 
Table 1 Demographic data of 70 patients

\begin{tabular}{lll}
\hline Parameters & PCV (35) & PSVPro (35) \\
\hline Age $(\mathrm{yr})$ & $4.8(1.6)$ & $4.9(1.7)$ \\
Range & $(3-8)$ & $(3-8)$ \\
Height $(\mathrm{cm})$ & $117.7(15.8)$ & $108.8(12.9)$ \\
Range & $(88-142)$ & $(88-135)$ \\
Weight $(\mathrm{kg})$ & $15.9(5.3)$ & $15.5(5.2)$ \\
Range & $(10-29)$ & $(10-30)$ \\
Type of surgery & Urethroplasty-34 & Urethroplasty-34 \\
& Urethroplasty+orchidopexy-1 orchidopexy-1 & $87.1(13.1)$ \\
Total surgical duration (min) & $88.2(13.6)$ & $(62-110)$ \\
& $(62-105)$ & $103.4(15.2)$ \\
Total duration of anesthesia time (min) & $105.5(15.5)$ & $(78-118)$ \\
Size of SAD & $(75-119)$ & $1 / 29 / 5$
\end{tabular}

Data expressed as mean (standard deviation) (range) or absolute numbers. SAD = supraglottic airway device

Table 2 Intraoperative parameters

\begin{tabular}{|c|c|c|c|c|}
\hline & PCV group & PSVPro group & Mean difference $(95 \% \mathrm{CI})$ & $P$ value \\
\hline Total consumption of propofol $\left(\mu \mathrm{g} \cdot \mathrm{kg}^{-1} \cdot \mathrm{min}^{-1}\right)$ & $166.2(20)$ & $132.9(17)$ & $33.3(24.2$ to 42.4$)$ & $<0.001$ \\
\hline Children having asynchronous events & $15(42.8)$ & 0 & & $<0.001$ \\
\hline Emergence time (min) & $10.1(1.7)$ & $6.6(1.3)$ & 3.5 (2.8 to 4.2$)$ & $<0.001$ \\
\hline Time to achieve modified Aldrete score $>9$ & $18.4(3.5)$ & $14.8(3.2)$ & $3.6(1.9$ to 5.2$)$ & $<0.001$ \\
\hline \multicolumn{5}{|l|}{$\mathrm{RE}$} \\
\hline T0 & $93(2.0)$ & $92.8(2.8)$ & $0.2(-1.0$ to 1.4$)$ & 0.05 \\
\hline $\mathrm{T} 1$ & $50.4(4.2)$ & $53.2(4.1)$ & $-2.8(-4.8$ to -0.8$)$ & 0.89 \\
\hline $\mathrm{T} 2$ & $48.7(4.7)$ & $49.6(4.5)$ & $-0.9(-3.1$ to 1.3$)$ & 0.80 \\
\hline $\mathrm{T} 3$ & $58.5(5.7)$ & $60(6.4)$ & $-1.5(-4.4$ to 1.4$)$ & 0.50 \\
\hline $\mathrm{T} 4$ & $93.3(2.2)$ & $93.7(2.4)$ & $-0.4(-1.5$ to 0.7$)$ & 0.61 \\
\hline \multicolumn{5}{|l|}{ SE } \\
\hline T0 & $88.3(2.0)$ & $88.1(2.4)$ & $0.2(-0.9$ to 1.3$)$ & 0.29 \\
\hline $\mathrm{T} 1$ & $46.1(3.6)$ & $47.9(4.3)$ & $-1.8(-3.7$ to 0.1$)$ & 0.31 \\
\hline $\mathrm{T} 2$ & $44.2(5.1)$ & $45.1(4.3)$ & $-0.9(-3.2$ to 1.4$)$ & 0.32 \\
\hline $\mathrm{T} 3$ & $55.8(5.6)$ & $55.6(5.8)$ & $0.2(-2.5$ to 2.9$)$ & 0.84 \\
\hline $\mathrm{T} 4$ & $88.6(1.8)$ & $88.5(1.9)$ & $0.1(-0.8$ to 1.0$)$ & 0.75 \\
\hline
\end{tabular}

Data expressed as mean (standard deviation and mean difference ( $95 \%$ confidence interval). $\mathrm{CI}=$ confidence interval. $\mathrm{RE}=$ response entropy; $\mathrm{SE}$ $=$ state entropy

$\mathrm{T} 0=$ baseline, $\mathrm{T} 1=10 \mathrm{~min}$ after anesthesia induction, $\mathrm{T} 2=$ at surgical incision, $\mathrm{T} 3=$ at stoppage of propofol infusion, $\mathrm{T} 4=$ when fully awake

Asynchronous events and oxygenation index were also analyzed. Fifteen children $(42.8 \%)$ in the PCV group had at least one episode of asynchronous ventilation.

There was no difference in the oxygenation index at the start of the case in both groups (mean difference, $-9.2 ; 95 \%$ CI, -39.1 to 20.6). Nevertheless, PSVPro mode significantly improved the oxygenation index at the end of surgery (mean difference, $-37.2 ; 95 \%$ CI, -61.2 to -13.2 ) (Table 3). There was no significant difference in the partial pressure carbon dioxide and $\mathrm{pH}$ at the end of surgery in both the groups.

There was no adverse event like LMA dislodgement, desaturation $<92 \%$, bronchospasm, or laryngospasm in any patient. 
Table 3 Arterial blood gas analysis

\begin{tabular}{|c|c|c|c|c|}
\hline Parameters & PCV & PSVPro & Mean difference $(95 \% \mathrm{CI})$ & $P$ value \\
\hline $\mathrm{PaO}_{2}(1)$ & $151.2(23.9)$ & $155.5(17.9)$ & $-4.6(-14.9$ to 6.9$)$ & 0.61 \\
\hline $\mathrm{PaO}_{2}(2)$ & $168(19.8)$ & $185.6(27.9)$ & $-17.6(-29.2$ to -6.1$)$ & 0.04 \\
\hline $\mathrm{PaCO}_{2}(1)$ & $39.6(3.2)$ & $41.6(3.2)$ & $-1.9(-3.4$ to -0.4$)$ & 0.94 \\
\hline $\mathrm{PaCO}_{2}(2)$ & $39.01(2.7)$ & $40.6(2.6)$ & $-1.5(-2.8$ to 0.2$)$ & 0.89 \\
\hline $\mathrm{pH}(1)$ & $7.33(0.003)$ & $7.31(0.02)$ & $0.02(0.01$ to 0.03$)$ & 0.62 \\
\hline $\mathrm{pH}(2)$ & $7.34(0.03)$ & $7.35(0.02)$ & $0.01(-0.03$ to -0.002$)$ & 0.55 \\
\hline Oxygenation index (1) & $372.7(61.3)$ & $381.9(63.8)$ & $-9.2(-39.1$ to 20.6$)$ & 0.81 \\
\hline Oxygenation index (2) & $419.5(58.1)$ & $456.4(41.1)$ & $-37.2(-61.2$ to -13.2$)$ & 0.04 \\
\hline
\end{tabular}

Data expressed as mean (standard deviation) $(95 \%$ confidence interval $[\mathrm{CI}]) .(1)=$ preoperative readings; $(2)=$ end of surgery readings. $P<0.05$ is considered significant. $\mathrm{PaO}_{2}=$ partial pressure of arterial oxygen; $\mathrm{PaCO}_{2}=$ partial pressure of $\mathrm{CO}_{2}$ in arterial blood

\section{Discussion}

The results of our study showed that the use of intraoperative PSVPro mode during GA in unparalyzed children decreases propofol consumption, emergence time and asynchrony events, and improves the oxygenation index.

There was almost $20 \%$ reduction in consumption of propofol in the PSV group. This might be elucidated by an improved ventilator-patient synchrony in the PSVPro mode which reduced work of breathing and eventually decreased anesthetic drug consumption. ${ }^{11}$ Our results are in accordance with Capdevila et al., ${ }^{10}$ who documented $28 \%$ reduction in propofol consumption in PSV mode compared with controlled mode of ventilation in adults. An additional advantage of PSV was an early emergence time, which can be explained by less propofol consumption in the PSVPro group. This decreased emergence time also translated to decreased time to achieve a modified Aldrete score of more than or equal to 9 in the pressure support group. Capdevila et al. observed a significant $15 \mathrm{~min}$ decrease in emergence time in the PSV group compared with the PCV group.

Capdevila et al. ${ }^{10}$ defined emergence time as the time to obtain a ten-point score on a five-question test. Such a questionnaire is not feasible in the pediatric population, therefore we relied on eye opening and following commands as simple criteria for emergence.

Asynchronies are a frequent issue in ventilated patients representing a mismatch between the inspiratory and expiratory times of patient and ventilator, and thus a failure to provide optimal ventilation. Patient-ventilator asynchrony was seen in $40 \%$ of patients in the PCV group. Asynchronous events were accompanied by a transient increase in entropy values which required escalation of propofol infusion. The entropy between both groups was kept at 40-60 in both groups by adjusting the propofol infusion rate. On the other hand, PSV mode provided pressure support to each breath initiated by the patient, thereby achieving an adequate tidal volume that was fully synchronized with the respiratory effort of the patient. This improved patient-ventilator synchrony and decreased work of breathing.

In our study, although the oxygenation index improved in both the groups, PSVPro resulted in significant better oxygenation at the end of surgery. Spontaneous breathing in PSVPro improved distribution of ventilation to dependent lung region and hence decreased ventilation perfusion matching. This fact has already been highlighted by previous authors who found improved oxygenation with the pressure support mode than with the spontaneous mode of breathing. ${ }^{12-14} \mathrm{~A}$ similar observation of improvement in lung functions and oxygenation with PSV compared with the PCV was made by Zoremba et al. in 68 moderately obese patients. ${ }^{15}$

Our study has some limitations. The major limitation of the study was the increased use of propofol to reduce asynchrony in the protocol, when propofol consumption was used as an outcome measure. Also, complete blinding was not possible. Another limitation was that although entropy was continuously monitored and propofol was titrated accordingly, to avoid data noise we recorded and analyzed the entropy values only at predefined time frames. Also, we did not measure time to discharge as it is the routine protocol in our institution to observe all patients for at least two hours postoperatively in the PACU. We did not compare it to patients on spontaneous ventilation as we had selected surgeries of more than $45 \mathrm{~min}$. These results are relevant only for patients receiving GA through the use of LMA. Also, these results cannot be extrapolated to very small children.

In conclusion, PSVPro mode decreases propofol consumption, emergence time and asynchrony events, and improves oxygenation index during GA under the LMA Proseal in unparalyzed anesthetized children. 
Author contributions Swapnabharati Moharana contributed to the acquisition, analysis, and interpretation of data and drafting the article. Divya Jain contributed to the conception and design of the study. Neerja Bhardwaj contributed to the study design and final editing. Komal Gandhi contributed to the analysis of data. Sandhya Yaddanapudi contributed to the interpretation of data. Badal Parikh contributed to the acquisition of data.

\section{Conflicts of interest None.}

Funding statement This research was carried out without funding.

Editorial responsibility This submission was handled by Dr. Gregory L. Bryson, Deputy Editor-in-Chief, Canadian Journal of Anesthesia.

\section{References}

1. Nouraei SA, Giussani DA, Howard DJ, Sandhu GS, Ferguson C, Patel A. Physiological comparison of spontaneous and positivepressure ventilation in laryngotracheal stenosis. $\mathrm{Br} \mathrm{J}$ Anaesth 2008; 101: 419-23.

2. Jagannathan $N$, Ramsey MA, White MC, Sohn L. An update on newer supraglottic airways with recommendations for clinical use. Paediatr Aanesth 2015; 75: 334-45.

3. Devitt JH, Wenstone R, Noel AG, O'Donnell MP. The laryngeal mask airway and positive-pressure ventilation. Anesthesiology 1994; 80: 550-5.

4. Keller C, Sparr HJ, Luger TJ, Brimacombe J. Patient outcomes with positive pressure versus spontaneous ventilation in nonparalysed adults with the laryngeal mask. Can J Anaesth 1998; 45: 564-7.

5. Goldmann K, Roettger $C$, Wulf $H$. Use of the ProSeal laryngeal mask airway for pressure-controlled ventilation with and without positive end-expiratory pressure in paediatric patients: a randomized, controlled study. Br J Anaesth 2005; 95: 831-4.

6. Jaber S, Tassaux D, Sebbane $M$, et al. Performance characteristics of five new anesthesia ventilators and four intensive care ventilators in pressure-support mode: a comparative bench study. Anesthesiology 2006; 105: 944-52.

7. Tokioka H, Nagano O, Ohta Y, Hirakawa M. Pressure support ventilation augments spontaneous breathing with improved thoracoabdominal synchrony in neonates with congenital heart disease. Anesth Analg 1997; 85: 789-93.

8. Christie JM, Smith RA. Pressure support ventilation decreases inspiratory work of breathing during general anesthesia and spontaneous ventilation. Anesth Analg 1992; 75: 167-71.

9. Jaber S, Sebbane M, Verzilli D, et al. Adaptive support and pressure support ventilation behavior in response to increased ventilator demand. Anesthesiology 2009; 110: 620-7.

10. Capdevila X, Jung B, Bernard N, Dadure C, Biboulet P, Jaber S. Effects of pressure support ventilation mode on emergence time and intra-operative ventilator function: a randomized controlled trial. PLoS One 2014; DOI: https://doi.org/10.1371/journal.pone. 0115139.

11. Garcia-Fernandez J, Tusman G, Suarez-Sipmann F, Llorens $J$, Soro $M$, Belda JF. Programming pressure support ventilation in pediatric patients in ambulatory surgery with a laryngeal mask airway. Anesth Analg 2007; 105: 1585-91.

12. Brimacombe $J$, Keller $C$, Hormann C. Pressure support ventilation versus continuous positive airway pressure with the laryngeal mask airway: a randomized crossover study of anesthetized adult patients. Anesthesiology 2000; 92: 1621-3.

13. Von Goedecke A, Brimacombe J, Hormann C, Jeske HC, Kleinsasser A, Keller C. Pressure support ventilation versus continuous positive airway pressure ventilation with proseal laryngeal mask airway: a randomized crossover study of anaesthetised pediatric patients. Anesth Analg 2005; 100: 357-60.

14. Lim B, Pawar D, Ng O. Pressure support ventilation vs spontaneous ventilation via ProSeal ${ }^{\mathrm{TM}}$ laryngeal mask airway in pediatric patients undergoing ambulatory surgery: a randomized controlled trial. Paediatr Anaesth 2012; 22: 360-4.

15. Zoremba M, Kalmus $G$, Dette $F$, Kuhn $C$, Wulf $H$. Effect of intraoperative pressure support vs pressure-controlled ventilation on oxygenation and lung function in moderately obese adults. Anesthesia 2010; 65: 124-9.

Publisher's Note Springer Nature remains neutral with regard to jurisdictional claims in published maps and institutional affiliations. 